

\title{
TEJIDO: Unidad en la diversidad
}

\section{WEAVING: Unity in Diversity}

\section{Pilar Flores}

\author{
ISSN (imp): 1390-4825 \\ ISSN (e): 2477-9199
}

\author{
Fecha de recepción: 19/03/18 \\ Fecha de aceptación: 06/05/18
}

\section{Resumen:}

La idea base de esta obra artística es crear comunidad bordando, incentivar comunidades cuyos miembros cultiven la atención y la observación de sí mismos y se encaminen hacia una convivencia pacífica en el reconocimiento de sí mismos y del otro. El proyecto TEJIDO es el resultado de la investigación en varias fuentes tanto de Oriente como de Occidente, toma la potencia de diversos pensamientos y se sustenta en prácticas antiguas. Esta obra es en sí misma un tejido, está en el cruce de diferentes maneras de ver el mundo, presenta una simultaneidad de geografías y tiempos.

\section{Pallabras clave:}

Comunidad, bordado, atención, convivencia pacífica, tejido, unidad, complementariedad, mito, símbolo

\section{Abstract:}

The core idea of this artistic work is to create community through embroidery, encourage communities whose members nurture attention and self-observation and move towards a peaceful coexistence in the recognition of themselves and the other. The project TEJIDO is a result of research in several sources, both from the East and the West. The power of different thoughts and ancient practices nurture this work. It is itself a weaving and a crossroads of different ways of seeing the world, presenting us a simultaneity of geographies and times.

\section{Key Words:}

communities, embroidery, attention, peaceful coexistence, weaving, unity, complementarity, myth, symbol

\section{Biografía de la autora:}

Pilar Flores. Quito, Ecuador, 1957. Artista Visual. Licenciada en Artes de la UCE (1981); Magister en Docencia Universitaria e Investigación Educativa por la PUCE (2005); autora de los libros Taller de Arte: una organización compleja, Trama (2006); Diario de un iris, Trama (2015); TEJIDO, PUCE (2017). Docente de la Facultad de Arquitectura, Diseño y Artes de la PUCE desde 1994. 
Con el paso del tiempo, el arte ha confirmado que no consiste solo en ese "medio para" a secas, o acaso ese objeto en su mismidad, sino en una metáfora en sí misma, de sí y de algo mayor, trascendente, pero al mismo tiempo exquisitamente cotidiano, pagano. Una de las expresiones artísticas universales que confirma mejor este principio es el tejido, su existencia, desarrollo y permanencia ulteriores, inmanentes a la condición y devenir humanos. En cualquier tejido sobresale ese carácter simbólico-alegórico per se, la pertinencia cósmica de sus texturas e irregularidades, la complejidad de sus bifurcaciones y madejas, la circunstancia irreversible de su instauración, así como la posibilidad de continuar estableciendo conexiones y patrones de infinita naturaleza y diversidad.

TEJIDO es un proyecto artístico trabajado a lo largo de muchos años, que partió de la noción de que la obra consiste también en la creación de comunidad o comunidades, bordando. El acto de bordar y tejer se convertía en una forma de incentivar a estas comunidades cuyos miembros cultivaran la atención y la observación de sí mismos, y se encaminaran hacia una convivencia pacífica en el "reconocimiento del otro como legítimo otro" (Maturana, 2007). En el proyecto TEJIDO me nutro de varias fuentes, tanto de Oriente como de Occidente, reconozco la potencia de diversos pensamientos y recurro a esas prácticas antiguas que sobreviven al paso del tiempo.

Esta obra constituye en sí misma un tejido, está situada en el cruce de diferentes maneras de ver el mundo, nos presenta una simultaneidad de geografías y tiempos que se entretejen; la obra se encuentra dentro de la obra y da lugar a esa especie de experiencia tautológica, pero especialmente profunda y diversa. Los bordados que realizamos se convirtieron en ese símbolo de nuestras conquistas (Botton, 2014:65), en la "alegoría humanizada", por llamarla de algún modo. Es por esta razón que el objetivo principal del proyecto fue la creación de estos espacios favorables donde las personas se expresaran y relacionaran. También es importante para este proyecto, la experiencia en la cotidianidad del tejido como lenguaje y como forma de escritura de la cultura andina, que tiene fundamentalmente una conciencia colectiva, elemento que ha inspirado, por ejemplo, los talleres trabajados con las mujeres de la parroquia de Cotogchoa, ubicada al sur occidente del cantón Rumiñahui, Ecuador. Ellas, siguiendo su tradición, cuentan su cotidiani- dad, sus labores, sus sueños y anhelos, a través de sus bordados (Ver figura 1).

Como consecuencia de este proceso de "comunión y expresión", TEJIDO aparece en la muestra Aliento. Antología de Pilar Flores, presentada en el Centro de Arte Contemporáneo, de Quito; así como el libro de artista $T E J I D O^{1}$, que recoge el proceso completo de la obra de la que forma parte también, presenta su registro, anota las ideas esenciales del desarrollo de los talleres, además, y lo que es muy importante, nombra a todos y cada uno de los participantes.

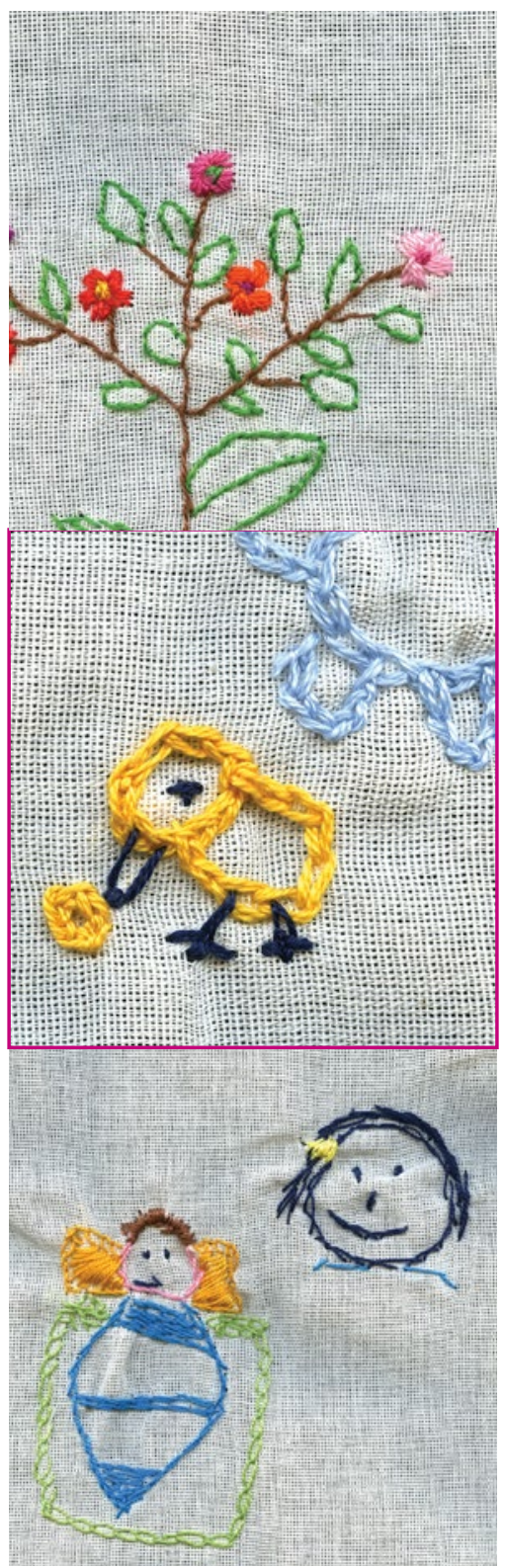

1: Bermello, V.; Puga, A.; Topón, P. (2015). [Bordados] Fuente: Flores, P. (2017). TEJIDO. Quito: Centro de Publicaciones PUCE.

1 Flores, P. (2017). TEJIDO. Quito: Centro de Publicaciones PUCE. 
El bordado es un ejercicio de atención, y cultivar la atención nos provee de muchos recursos. La atención puede dirigirse hacia el interior (como autoconocimiento), hacia los demás (con una mejora de nuestras relaciones) o hacia el exterior, es decir, hacia el cosmos en general (lo que nos permite navegar por el mundo). (Goleman, 2013). Esa es la apuesta, seres humanos con más habilidades para la vida. Lo he experimentado en mi práctica del yoga, donde la atención es fundamental. Desde hace 44 años me introduje en la experiencia del Raja Yoga, conocido en Occidente a través de las enseñanzas de Patanjali, quien posiblemente en el siglo III a. C. escribió en sánscrito los textos Yoga sūtras, el libro más antiguo sobre esta ciencia. Desde ese momento me comprometí con la práctica meditativa como una forma de vida y en 1994 abrí mi casa para compartir esta práctica con una comunidad de meditadores, que se mantiene vigente hasta el presente. Siguieron años en los que arte y meditación se combinaron, se entretejieron, se enriquecieron; dando lugar a la obra Cartografía Interior, presentada en Quito, Arte Actual de la Facultad Latinoamericana de Ciencias Sociales (Flacso), en el 2012. Esta constituyó un estudio gráfico de los Yoga sütras de Patanjali y del Bhagavad-gìtā.

Desde inicios de los años 90 realizo talleres artísticos en sectores rurales, pero es en el 2000 que me parecía que estaba lista para compartir estas vivencias en las que se conjugan el arte con prácticas de atención y aquietamiento. Así, acojo en mi taller a hombres y mujeres deseosos de conocerse mejor y abordar las complejidades de su propio ser a través de procesos artísticos. Propongo crear juntos, a partir de una pregunta generadora de un proceso interno, lo que he llamado Encuentros de creación desde el silencio, actos artísticos y poéticos llevados a cabo semanalmente, a lo largo de 15 años.

Fruto de estos intercambios y experiencias, de los talleres, de mi trabajo con niños y niñas en las escuelas fiscales del programa Arteducarte (20002005), de mis clases en la PUCE (desde 1994), de la propuesta Taller de arte, una organización compleja (2006), nace en el 2007 una obra titulada Palimpsesto, presentada en el Museo de la Ciudad, en Quito. Aquí se conjugaron todos estos elementos a manera de ritual, y además se inspiró en un poema $^{2}$ de Cecilia Velasco.

2 ...un tráfico dulce recorre/ sin pausas/ toda la intimidad/ y el alma se hace visible/ como un hilo rojo...
Así, invité a mujeres de distintas geografías a bordar en silencio, con un hilo rojo, un hilo como el que Ariadna le ofrece a Teseo en la Creta antigua, para que consiguiese salir del laberinto y escapar del Minotauro. Palimpsesto se construyó multidimensionalmente como un rizoma. Fue creciendo como un sistema abierto, en la combinación de mito, símbolo, rito y fiesta.

La obra TEJIDO es la expansión y potencialización de esa idea. En el 2007, noventa y seis bordados constituían el centro de un laberinto (Palimpsesto), pero en el 2015 trescientos bordados crearon una gran red de comunión y descubrimiento. Se fue tejiendo en las relaciones y en la articulación de diferentes intensidades, en las que finalmente se

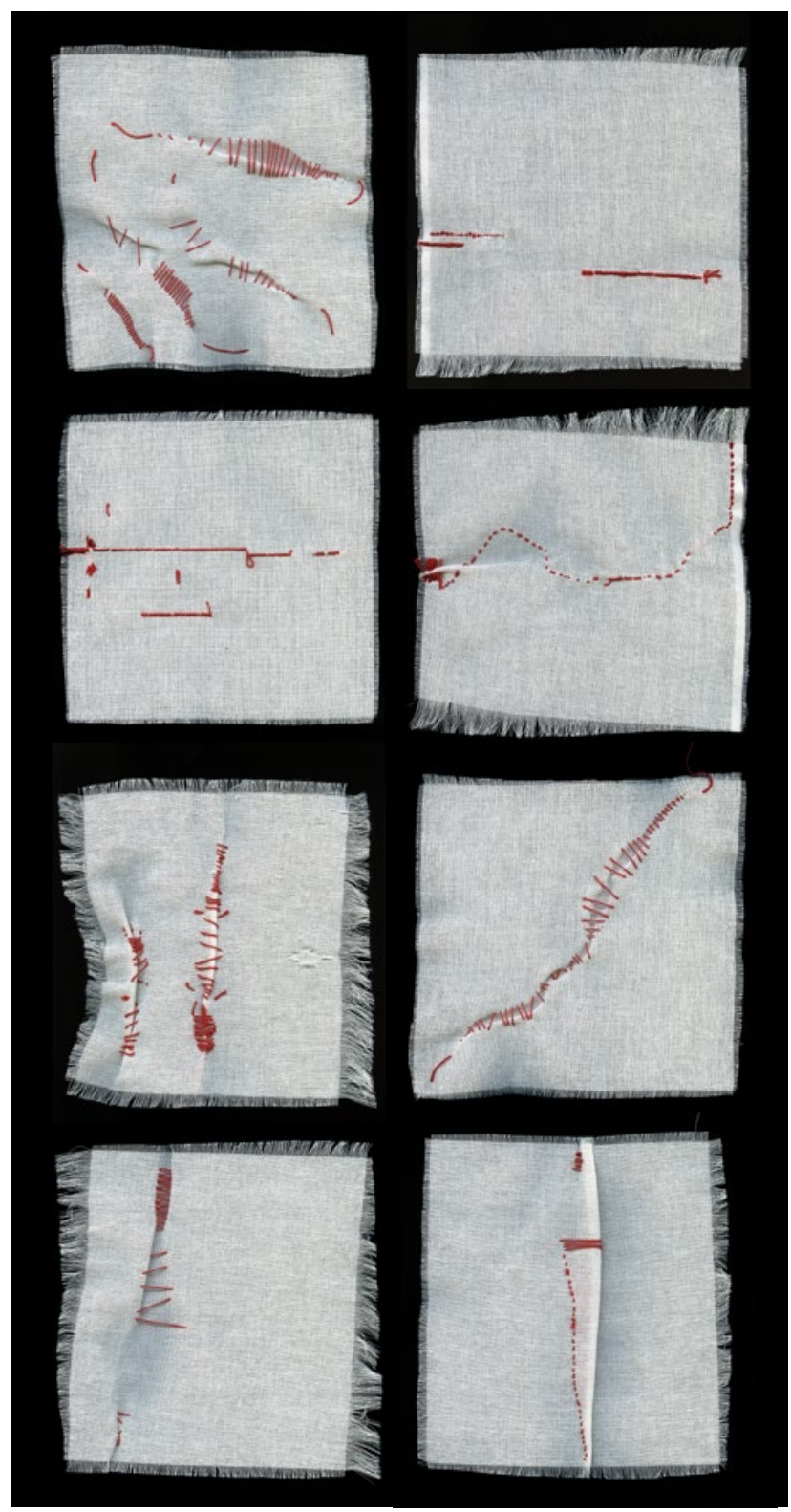

2: Calero, S. (2015). [Bordados] Fuente: Flores, P. (2017). TEJIDO. Quito:

Centro de Publicaciones PUCE. 
buscó el equilibrio dinámico de los extremos (por ejemplo, la fuerza de lo femenino integrada a lo masculino como su complementario). (Ver figura 2)

A través del mito accedemos al símbolo. El mito es la "experiencia de una vida remota intemporal, cargada de significados que iluminan el presente. El mito es un símbolo en palabras (...) y una exploración simbólica del misterio del hombre en el cosmos" (Rueda, 1993). O, tal como dice Campbell (1991), los mitos son pistas de las potencialidades espirituales de la vida humana. En el núcleo de esta obra están dos preguntas: ¿Qué le ayuda al ser humano a actuar desde su fuerza interna? ¿Cómo impactan sus acciones en la comunidad en la que, vive, la que en todos los casos, es una comunidad diversa?

Hay tradiciones antiguas, sobre todo orientales, que nos describen el universo como si estuviese sostenido por una red, en la cual el todo y las partes están interconectados en un movimiento continuo. Cada uno de nosotros es un nodo de este tejido, cada acción tiene una repercusión y provoca que toda la red se estremezca. "Intersomos" dice la maestra zen Bertha Meneses (2012), mientras que el monje budista Thich Naht Hanh, activista por la paz, termina acuñando la expresión "Interexistencia" (McLeod, 2010) para referirse a este sentido de "relacionalidad", a la comprensión de la vida como un todo, físico, pero también metafísico. Esto último encuentra su mejor alegoría en la vida cotidiana y en la forma en que se establecen los colectivos, comunidades y cualquier lazo fraternal, donde se tiene como objetivo la instauración de esta totalidad, que contiene en sí misma nuestras semejanzas y diferencias. Lo ha dicho con toda precisión y belleza el erudito hindú Ananda K. Coomaraswamy:

Hay así una incesante multiplicación del Uno inagotable, y una unificación de los indefinidamente Muchos. Tales son los comienzos y los finales de los mundos y de los seres individuales: expandidos desde un punto sin posición ni dimensiones, y desde un ahora sin fecha ni duración, cumplen su destino, y

3 "Para la filosofía andina, el individuo como tal es un "nada" (un "no-ente"), es algo totalmente perdido, si no se halla insertado en una red de múltiples relaciones” Estermann, J. (2006). Filosofía Andina. La Paz: Colección "Teología y Filosofía Andinas". p. 110.

4 El término griego holon, que significa todo. cuando acaba su tiempo retornan a «casa», al Mar en el que se originó su vida. (Coomaraswamy, s.f.: 14)

Por su parte, la cosmovisión andina ${ }^{3}$ coincide con Oriente en lo que el filósofo y teólogo Josef Estermann (2006) ha denominado como "principio de relacionalidad", que a su vez se encuentra fundamentado en la correspondencia (que todo armoniza) y la complementariedad (que todo se completa):

(...) que todo está de una u otra manera relacionado (vinculado, conectado) con todo. (...) la entidad básica no es el 'ente' sustancial, sino la relación; por lo tanto, para la filosofía andina, no es que los entes particulares, adicionalmente a su existencia particular, se relacionan en un segundo momento y llegan a formar un 'todo integral' (holon ${ }^{4}$ ), una red de interrelaciones y conexiones. $\mathrm{Al}$ contrario: recién en base a la primordialidad de esta estructura relacional, los entes particulares se constituyen como 'entes'. (Estermann, 2006: 126)

Esto le permite llegar a la conclusión de que la "lógica andina" tiene mucho más en común con la "lógica oriental", poniendo a su vez sobre la palestra la idea de que la "lógica occidental" se habría autoproclamado como una especie de supra-paradigma y acaparado para sí las disciplinas de la "filosofía” y "estética”. Pero en el proyecto que estoy presentando he pretendido trabajar con aquellas nociones que nos conectan, no constituye mi intención abordar las cuestiones que nos diferencian en cuanto a lógicas diversas. Se trata más bien de un modelo "filosófico intercultural", a decir de Esterman (2006) y por extensión de una propuesta intercultural fundamentada en una "estética intercultural”, a modo de paráfrasis. Esto es, que cada integrante de la red tiene una fortaleza interior que sostiene el tejido completo, que somos diferentes y la diversidad nutre estos principios comunes.

Es por todo esto que se ha vuelto recurrente para mí la idea de Bertha Meneses, aquello de que está a punto de emerger una nueva forma de pensar, un cambio de paradigma, una transformación de las sociedades: el paso de una vida desarrollada desde la competencia a la vida sostenida desde la colaboración, la reciprocidad, el trabajo comunitario. En este sentido, se comienza a intuir una nueva forma 


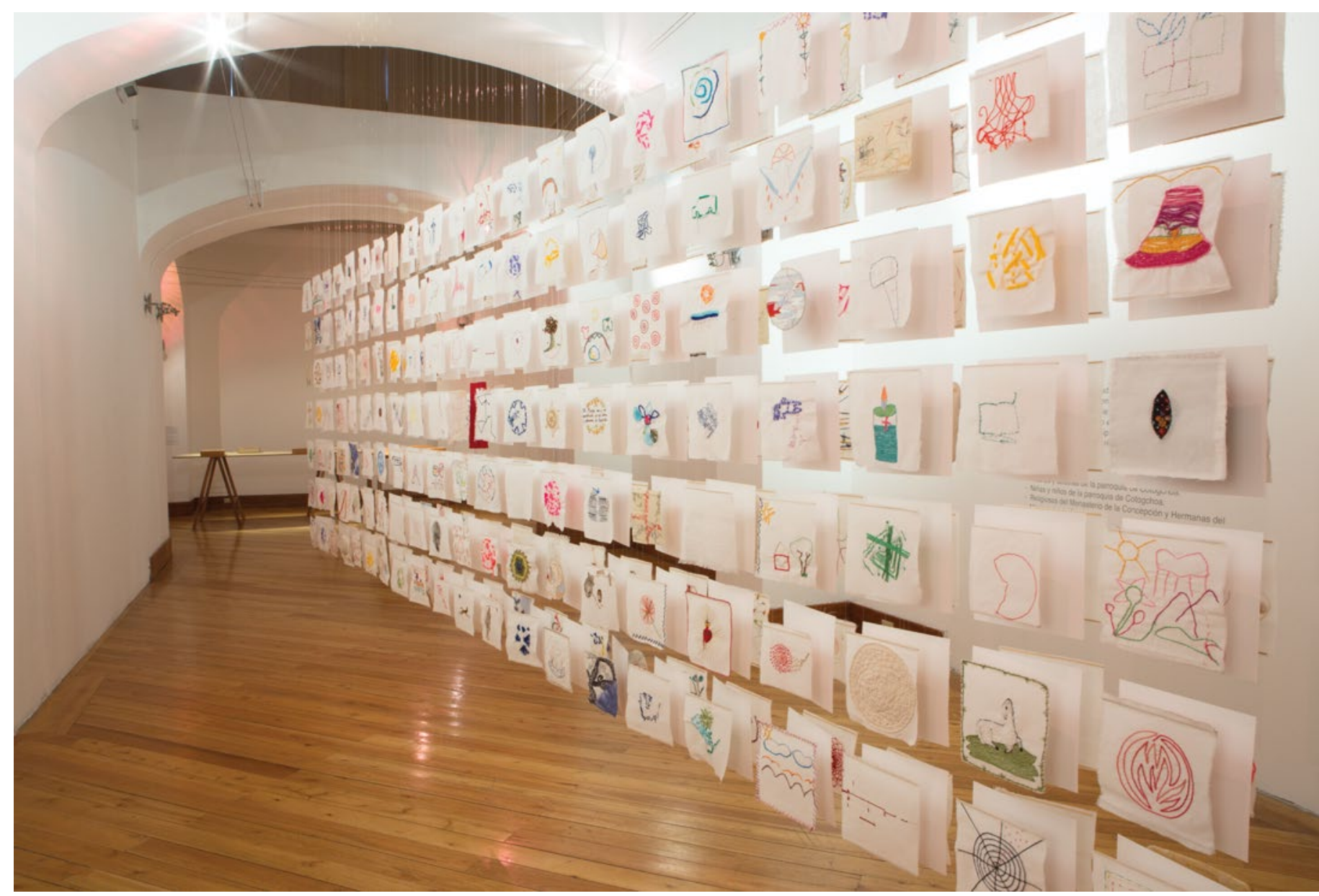

3. TEJIDO, Aliento. Antología de Pilar Flores. (2015-2016).

Centro de Arte Contemporáneo, Quito.

[Fotografía] Fuente: Vargas M., G.

de resolver y enfrentar los problemas. Existen algunos proyectos emancipadores donde se concibe la política no como ese juego tradicional del poder y la lucha por quién tiene este poder, sino de una "nueva política", a decir de Melvin McLeod, como una de las formas más importantes que tenemos los seres humanos para vivir juntos y alcanzar objetivos colectivos (2010).

Cada ser que forma parte de las comunidades que tejen esta red en la obra TEJIDO (Ver Imagen 3) fue invitado a bordar en conexión con su fortaleza interior, en un trabajo de presencia consciente, de identificación y valoración de su propio poder interno y del poder colectivo. La Paz es Poder (Peace is Power) escribe en una de sus obras la artista japonesa Yoko Ono (MOMA, NY, 2017). Es por esto que con "presencia consciente" me refiero más bien a una actitud de paz, de serenidad, de alerta, a una actitud meditativa. Reginald A. Ray afirma que la meditación es en sí misma una acción política radical, cuyo objetivo fundamental es cambiar el mundo, y enfatiza que Gandhi fue un ejemplo de lo poderosa que es la libertad interior adquirida en la meditación, para el mundo de la política (Ray, 2010). La práctica meditativa conduce al "ego a abandonar la escena y cuando esto ocurre, descubrimos una 'mente' interior que es inmensa, no tiene miedo y posee sorprendentes recursos", agrega Ray (2010: 95). En fin, que con Gandhi y la resistencia no violenta, el mundo tiene la prueba de que hay otras alternativas para resolver los conflictos.

Así, alguien que experimenta el sentido profundo de la meditación es un ser pacífico, solidario, que reconoce la interdependencia en una sociedad que potencializa la riqueza humana y cultural, ajena a posiciones excluyentes. Alguien que ejercita su atención en la construcción de una mejor calidad de vida actuaría a partir de su práctica, con serenidad, consistencia y fuerza, en el contexto de la comunidad a la que pertenece, conocedor de sus necesidades y de la responsabilidad que tiene en ella.

Desde esta práctica, para emprender cualquier actividad externa, primero está el compromiso de trabajar en sí mismo. El compromiso consiste en 


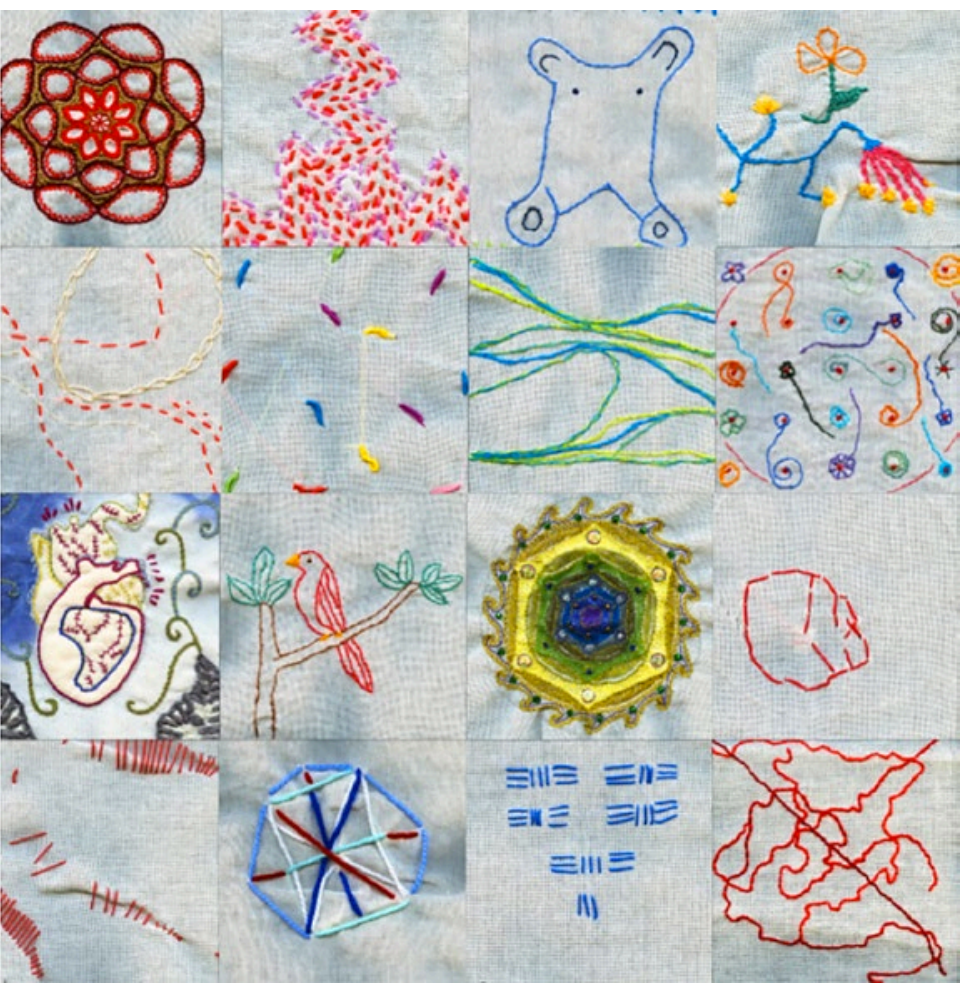

4. Flores, P. (2017). TEJIDO. Quito: Centro de Publicaciones PUCE. [Bordados varios autores].

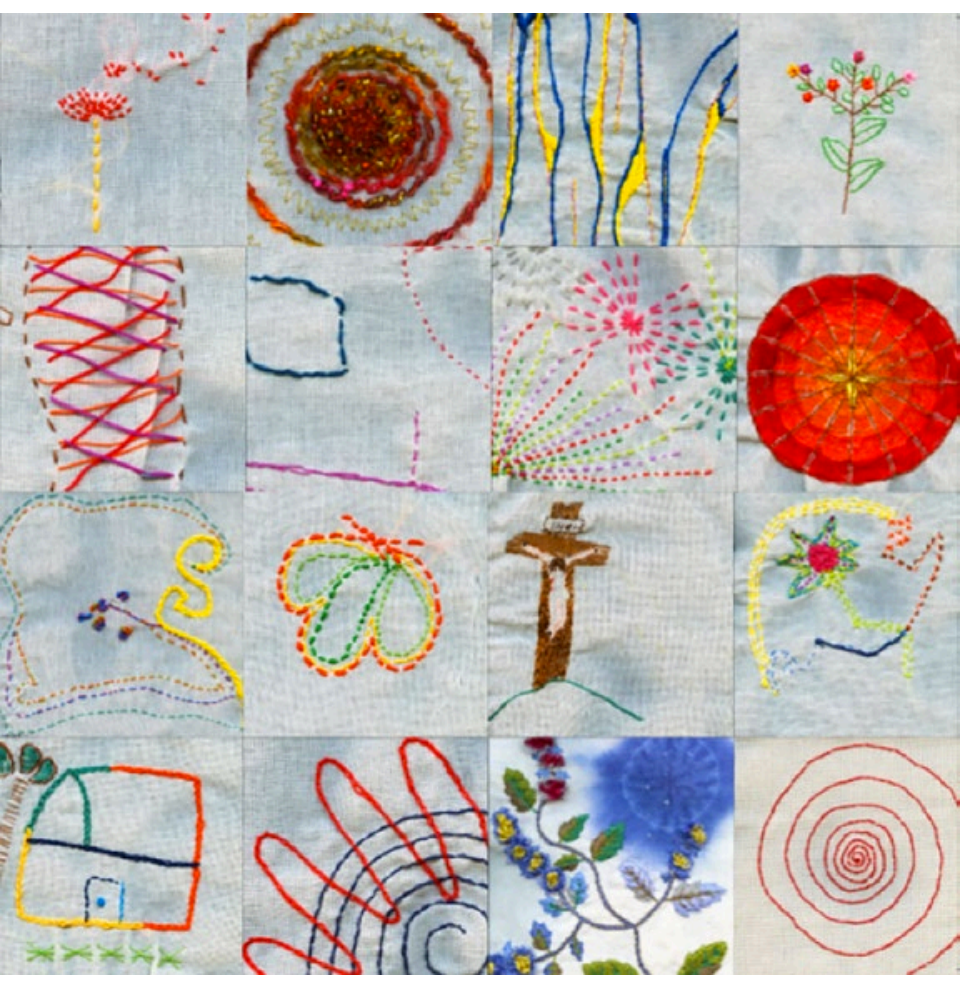

5. Flores, P. (2017). TEJIDO. Quito: Centro de Publicaciones PUCE. [Bordados varios autores]

actuar con apertura y escucha, actuar con respeto y empatía, actuar con paz y aceptación de la diversidad, actuar desde la fuerza interna. Esta es la propuesta de la obra TEJIDO: la convivencia pacífica y solidaria desde el cultivo de la atención en las fuerzas que nos construyen como seres humanos y que nos llevan a alcanzar libertad y plenitud para el bien de la comunidad de la que formamos parte.

El rito se asocia con un viaje interior; así aconteció en la trayectoria de cada uno de los participantes, contactándose con su "fuerza interior", respondiendo a la invitación inicial. Y la fiesta es la celebración colectiva y el encuentro. En la obra TEJIDO, cada bordado es el símbolo de una vida: un adulto, un niño, una niña, unidos a los demás desde su propia fuerza para el beneficio de toda la red que nos configura (Ver figuras 4 y 5). Aquí se aborda de manera fundamental el tema de la comunidad como una inmensa red de reciprocidad y de responsabilidades compartidas, el desafío prioritario que hoy tenemos los seres humanos de ser solidarios con nuestra propia especie ${ }^{5}$. Martin Luther King dice que todas las cosas existen en una red de reciprocidad, en la que todo cuanto le afecta a uno directamente, afecta a todos indirectamente (Johnson, 2010). En esta red de vínculos, cada ser humano, cada uno de nosotros, somos un nodo y un pensamiento, un acto, un movimiento que repercute invariablemente en la red entera.

\section{BIBLIOGRAFÍA:}

Botton, A., Armstrong, J. (2014). El arte como terapia. México D.F: Editorial Océano.

Campbell, J. (1991). El poder del mito. En diálogo con Bill Moyers. Barcelona, España: Emecé.

Coomaraswamy, A. K. (s. f.). Hinduismo $y$ Budismo. Buenos Aires, Argentina www. infotematica.com.ar

Estermann, J. (2006). Filosofía Andina. La Paz: Colección "Teología y Filosofía Andinas".

Flores, P. (2017). TEJIDO. Quito, Ecuador: Centro de Publicaciones PUCE.

Goleman, D. (2013). Focus: Desarrolla la atención para alcanzar la excelencia. Barcelona, España: Kairos S.A.

Johnson, C. R. (2010). 2. Encarnar la paz: Principios de la política con conciencia. En M. McLeod (Ed.), Politica con conciencia. p. 83-96). Barcelona, España: Kairós, S. A.

Maturana, H. (2007). Transformación en la Con-

Jaime Costales Peñaherrera. Entrevista en Radio Visión Quito (91.7) desde Buenos Días con Diego Oquendo, 14 de noviembre de 2017. 
vivencia. Santiago de Chile, Chile: J. C. Sáez Editor.

McLeod, M. (2010). Politica con conciencia. Barcelona , España: Editorial Kairos, S.A.

Meneses, B. (2012). El Zen un camino de sabiduría y Compasión. La caja de Pandora. Recuperado de http://www.lacajadepandora.eu/2013/04/ el-zen-un-camino-de-sabiduria-y-compasion-berta-meneses-maestra-zen/

Ray, R. A. (2010). 6. La política del Buddha. En McLeod, M. (Ed.), Politica con conciencia. (1st ed., pp. 83-96). Barcelona: Kairós, S. A.

Rueda M. V. (1993). Mitología. Cuadernos de Antropología,1. Quito, Ecuador: EdiPuce. 